\title{
Correction to: Safety and efficacy of guanfacine extended-release in adults with attention-deficit/hyperactivity disorder: an open-label, long-term, phase 3 extension study
}

${\text { Akira Iwanami }{ }^{1 *}, \text { Kazuhiko Saito }^{2} \text {, Masakazu Fujiwara }}^{3}$, Daiki Okutsu ${ }^{4}$ and Hironobu Ichikawa ${ }^{5}$

Correction to: BMC Psychiatry 20, 485 (2020)

https://doi.org/10.1186/s12888-020-02867-8

Following publication of the original article [1], the authors identified an error in Table 4. The correct table is given below.

The author group has been updated above and the original article [1] has been corrected.

\section{Author details}

'Department of Psychiatry, Showa University School of Medicine, 6-11-11 Kita Karasuyama, Setagaya-ku, Tokyo 157-8577, Japan. ${ }^{2}$ Aiiku Counselling Office, Aiiku Research Institute, Imperial Gift Foundation Boshi-Aiiku-Kai, Tokyo, Japan. ${ }^{3}$ Biostatistics Center, Shionogi \& Co., Ltd., Osaka, Japan. ${ }^{4}$ Clinical

Research Department, Shionogi \& Co., Ltd, Osaka, Japan. ${ }^{5}$ Japan Developmental Disorders Network, Tokyo, Japan.

Published online: 22 December 2020

\section{Reference}

1. Iwanami A, et al. Safety and efficacy of guanfacine extended-release in adults with attention-deficit/hyperactivity disorder: an open-label, long-term, phase 3 extension study. BMC Psychiatry. 2020;20:485. https://doi.org/10. 1186/s12888-020-02867-8.

The original article can be found online at https://doi.org/10.1186/s12888020-02867-8

* Correspondence: iwanami@med.showa-u.ac.jp

'Department of Psychiatry, Showa University School of Medicine, 6-11-11 Kita Karasuyama, Setagaya-ku, Tokyo 157-8577, Japan

Full list of author information is available at the end of the article

(c) The Author(s). 2020 Open Access This article is licensed under a Creative Commons Attribution 4.0 International License, which permits use, sharing, adaptation, distribution and reproduction in any medium or format, as long as you give appropriate credit to the original author(s) and the source, provide a link to the Creative Commons licence, and indicate if changes were made. The images or other third party material in this article are included in the article's Creative Commons licence, unless indicated otherwise in a credit line to the material. If material is not included in the article's Creative Commons licence and your intended use is not permitted by statutory regulation or exceeds the permitted use, you will need to obtain permission directly from the copyright holder. To view a copy of this licence, visit http://creativecommons.org/licenses/by/4.0/ The Creative Commons Public Domain Dedication waiver (http://creativecommons.org/publicdomain/zero/1.0/) applies to the data made available in this article, unless otherwise stated in a credit line to the data. 
Table 4 Key Efficacy Measures During Long-term Treatment With GXR

\begin{tabular}{|c|c|c|c|c|c|c|}
\hline \multirow[b]{2}{*}{ Endpoint } & \multirow[b]{2}{*}{ Patient populations } & \multirow[b]{2}{*}{ Week 0} & \multicolumn{2}{|l|}{ Week 50} & \multicolumn{2}{|c|}{$\begin{array}{l}\text { Last observation in the } \\
\text { treatment period }\end{array}$} \\
\hline & & & $\begin{array}{l}\text { Change from } \\
\text { week } 0\end{array}$ & $p$-value & $\begin{array}{l}\text { Change from } \\
\text { week } 0\end{array}$ & $p$-value \\
\hline \multicolumn{7}{|l|}{ ADHD-RS-IV ${ }^{\mathrm{a}}$, mean $(95 \% \mathrm{Cl})$} \\
\hline \multirow[t]{3}{*}{ Total scores } & Former placebo patients & $24.76(22.53,26.99)$ & $-8.31(-10.72,-5.89)$ & $<.0001$ & $-5.94(-7.53,-4.36)$ & $<.0001$ \\
\hline & Former GXR patients & $22.31(19.65,24.97)$ & $-9.11(-11.19,-7.03)$ & $<.0001$ & $-7.87(-9.68,-6.06)$ & $<.0001$ \\
\hline & New patients & $32.80(30.93,34.68)$ & $-19.69(-23.35,-16.03)$ & $<.0001$ & $-16.54(-19.77,-13.31)$ & $<.0001$ \\
\hline \multirow[t]{3}{*}{ Inattention score } & Former placebo patients & $17.36(15.97,18.76)$ & $-5.51(-7.15,-3.87)$ & $<.0001$ & $-3.90(-5.03,-2.76)$ & $<.0001$ \\
\hline & Former GXR patients & $15.37(13.68,17.07)$ & $-5.82(-7.22,-4.42)$ & $<.0001$ & $-4.87(-6.04,-3.70)$ & $<.0001$ \\
\hline & New patients & $21.68(20.12,23.24)$ & $-12.10(-14.70,-9.51)$ & $<.0001$ & $-10.02(-12.28,-7.76)$ & $<.0001$ \\
\hline \multirow[t]{3}{*}{ Hyperactivity-impulsivity score } & Former placebo patients & $7.40(6.15,8.65)$ & $-2.80(-4.00,-1.59)$ & $<.0001$ & $-2.05(-2.77,-1.32)$ & $<.0001$ \\
\hline & Former GXR patients & $6.94(5.55,8.32)$ & $-3.29(-4.40,-2.17)$ & $<.0001$ & $-3.00(-3.96,-2.04)$ & $<.0001$ \\
\hline & New patients & $11.12(9.50,12.74)$ & $-7.59(-9.81,-5.36)$ & $<.0001$ & $-6.51(-8.25,-4.78)$ & $<.0001$ \\
\hline \multicolumn{7}{|l|}{ CAARS scores (DSM-M $)^{a}$, mean (95\% Cl) } \\
\hline \multirow[t]{3}{*}{ Total ADHD symptoms } & Former placebo patients & $25.08(22.93,27.23)$ & $-6.27(-8.65,-3.89)$ & $<.0001$ & $-4.60(-6.17,-3.02)$ & $<.0001$ \\
\hline & Former GXR patients & $22.74(20.07,25.42)$ & $-8.38(-10.90,-5.86)$ & $<.0001$ & $-7.30(-9.49,-5.10)$ & $<.0001$ \\
\hline & New patients & $31.32(28.64,33.99)$ & $-17.31(-20.89,-13.73)$ & $<.0001$ & $-15.08(-18.49,-11.66)$ & $<.0001$ \\
\hline \multirow[t]{3}{*}{ Inattentive symptoms } & Former placebo patients & $17.40(16.14,18.65)$ & $-3.96(-5.57,-2.35)$ & $<.0001$ & $-2.90(-4.02,-1.79)$ & $<.0001$ \\
\hline & Former GXR patients & $15.55(13.90,17.19)$ & $-5.40(-7.03,-3.77)$ & $<.0001$ & $-4.51(-5.89,-3.13)$ & $<.0001$ \\
\hline & New patients & $20.39(18.48,22.30)$ & $-11.00(-13.54,-8.46)$ & $<.0001$ & $-9.15(-11.47,-6.83)$ & $<.0001$ \\
\hline \multirow[t]{3}{*}{ Hyperactive-impulsive symptoms } & Former placebo patients & $7.68(6.38,8.98)$ & $-2.31(-3.55,-1.06)$ & .0005 & $-1.69(-2.47,-0.92)$ & $<.0001$ \\
\hline & Former GXR patients & $7.19(5.79,8.60)$ & $-2.98(-4.30,-1.65)$ & $<.0001$ & $-2.79(-3.92,-1.66)$ & $<.0001$ \\
\hline & New patients & $10.93(9.36,12.49)$ & $-6.31(-8.17,-4.45)$ & $<.0001$ & $-5.93(-7.56,-4.29)$ & $<.0001$ \\
\hline \multicolumn{7}{|c|}{ CGI-I response rates ${ }^{\mathrm{b}}, \%$ of patients $(95 \% \mathrm{Cl})$} \\
\hline \multirow[t]{3}{*}{ Improvement rate (disease scores 1 or 2) } & Former placebo patients & $3.4(0.7,9.6)^{c}$ & $51.0(36.3,65.6)$ & NA & $35.2(25.3,46.1)$ & NA \\
\hline & Former GXR patients & $4.8(1.0,13.5)^{c}$ & $64.4(48.8,78.1)$ & NA & $53.2(40.1,66.0)$ & NA \\
\hline & New patients & $0.0(0.0,8.6)^{c}$ & $79.3(60.3,92.0)$ & NA & $65.9(49.4,79.9)$ & NA \\
\hline \multicolumn{7}{|c|}{ PGI-I response rates ${ }^{b}, \%$ of patients $(95 \% \mathrm{Cl})$} \\
\hline \multirow[t]{3}{*}{ Improvement rate (disease scores 1 or 2) } & Former placebo patients & $8.0(3.3,15.7)^{c}$ & $28.6(16.6,43.3)$ & NA & $19.3(11.7,29.1)$ & NA \\
\hline & Former GXR patients & $9.7(3.6,19.9)^{c}$ & $42.2(27.7,57.8)$ & NA & $33.9(22.3,47.0)$ & NA \\
\hline & New patients & $9.8(2.7,23.1)^{c}$ & $37.9(20.7,57.5)$ & NA & $31.7(18.1,48.1)$ & NA \\
\hline \multicolumn{7}{|c|}{ Patients not ill or borderline mentally ill ${ }^{\mathrm{b}}, \%$ of patients $(95 \% \mathrm{Cl})$} \\
\hline \multirow[t]{3}{*}{ CGI-S scores 1 or 2} & Former placebo patients & $0.0(0.0,4.1)$ & $14.3(5.9,27.2)$ & NA & $8.0(3.3,15.7)$ & NA \\
\hline & Former GXR patients & $0.0(0.0,5.8)$ & $26.7(14.6,41.9)$ & NA & $22.6(12.9,35.0)$ & NA \\
\hline & New patients & $0.0(0.0,8.6)$ & $20.7(8.0,39.7)$ & NA & $17.1(7.2,32.1)$ & NA \\
\hline \multicolumn{7}{|l|}{ AAQoL $^{a}$, mean $(95 \% \mathrm{Cl})$} \\
\hline \multirow[t]{3}{*}{ Total score } & Former placebo patients & $46.43(43.21,49.64)$ & $4.13(0.50,7.75)$ & .0266 & $2.81(0.31,5.30)$ & .0282 \\
\hline & Former GXR patients & $54.27(49.78,58.77)$ & $4.29(0.35,8.23)$ & .0334 & $4.04(0.88,7.20)$ & .0131 \\
\hline & New patients & $43.28(38.38,48.17)$ & $12.75(6.68,18.81)$ & .0002 & $9.22(4.11,14.34)$ & .0008 \\
\hline \multirow[t]{3}{*}{ Life productivity } & Former placebo patients & $48.04(43.75,52.33)$ & $2.64(-3.32,8.61)$ & .3775 & $2.89(-0.94,6.72)$ & .1377 \\
\hline & Former GXR patients & $57.88(52.69,63.08)$ & $8.74(4.69,12.79)$ & $<.0001$ & $8.08(4.76,11.41)$ & $<.0001$ \\
\hline & New patients & $44.29(37.72,50.86)$ & $17.08(9.11,25.06)$ & .0001 & $14.38(7.75,21.00)$ & $<.0001$ \\
\hline
\end{tabular}


Table 4 Key Efficacy Measures During Long-term Treatment With GXR (Continued)

\begin{tabular}{|c|c|c|c|c|c|c|}
\hline \multirow[b]{2}{*}{ Endpoint } & \multirow[b]{2}{*}{ Patient populations } & \multirow[b]{2}{*}{ Week 0} & \multicolumn{2}{|l|}{ Week 50} & \multicolumn{2}{|c|}{$\begin{array}{l}\text { Last observation in the } \\
\text { treatment period }\end{array}$} \\
\hline & & & $\begin{array}{l}\text { Change from } \\
\text { week } 0\end{array}$ & $p$-value & $\begin{array}{l}\text { Change from } \\
\text { week } 0\end{array}$ & $p$-value \\
\hline \multirow[t]{3}{*}{ Psychological health } & Former placebo patients & $47.02(42.20,51.83)$ & $5.27(0.57,9.97)$ & .0286 & $2.60(-1.14,6.34)$ & .1710 \\
\hline & Former GXR patients & $54.91(48.88,60.93)$ & $2.78(-2.69,8.25)$ & .3117 & $1.57(-2.82,5.96)$ & .4771 \\
\hline & New patients & $43.39(36.92,49.86)$ & $11.35(4.29,18.41)$ & .0027 & $5.62(-0.57,11.82)$ & .0739 \\
\hline \multirow[t]{3}{*}{ Life outlook } & Former placebo patients & $41.93(38.20,45.66)$ & $2.59(-1.97,7.15)$ & .2597 & $1.37(-1.76,4.51)$ & .3868 \\
\hline & Former GXR patients & $46.10(41.64,50.56)$ & $-1.90(-6.44,2.63)$ & .4016 & $-0.35(-4.08,3.37)$ & .8510 \\
\hline & New patients & $40.17(35.10,45.24)$ & $8.23(1.25,15.21)$ & .0225 & $6.06(0.71,11.41)$ & .0275 \\
\hline \multirow[t]{3}{*}{ Relationships } & Former placebo patients & $48.47(44.01,52.92)$ & $8.16(2.70,13.63)$ & .0042 & $4.88(1.15,8.61)$ & .0109 \\
\hline & Former GXR patients & $57.02(50.97,63.06)$ & $5.00(-1.10,11.10)$ & .1058 & $4.26(-0.82,9.34)$ & .0984 \\
\hline & New patients & $45.24(38.72,51.77)$ & $11.21(3.26,19.16)$ & .0074 & $6.63(-0.12,13.37)$ & .0542 \\
\hline \multicolumn{7}{|l|}{ BRIEF-A T-score ${ }^{a}$, mean $(95 \%$ Cl) } \\
\hline \multirow[t]{3}{*}{ Inhibit } & Former placebo patients & $57.24(54.81,59.66)$ & $-3.69(-6.39,-0.99)$ & .0084 & $-2.39(-4.15,-0.63)$ & .0084 \\
\hline & Former GXR patients & $51.68(49.20,54.16)$ & $-1.84(-4.04,0.35)$ & .0977 & $-2.66(-4.65,-0.66)$ & .0098 \\
\hline & New patients & $59.68(56.49,62.87)$ & $-8.07(-11.76,-4.38)$ & .0001 & $-8.25(-11.28,-5.22)$ & $<.0001$ \\
\hline \multirow[t]{3}{*}{ Shift } & Former placebo patients & $69.55(66.83,72.27)$ & $-5.84(-9.00,-2.68)$ & .0005 & $-3.29(-5.51,-1.08)$ & .0040 \\
\hline & Former GXR patients & $62.73(59.04,66.41)$ & $-4.60(-8.30,-0.90)$ & .0159 & $-3.70(-6.57,-0.84)$ & .0121 \\
\hline & New patients & $70.07(66.33,73.81)$ & $-8.86(-11.81,-5.91)$ & $<.0001$ & $-8.63(-11.18,-6.07)$ & $<.0001$ \\
\hline \multirow[t]{3}{*}{ Emotional control } & Former placebo patients & $57.80(55.51,60.08)$ & $-4.39(-7.55,-1.23)$ & .0075 & $-3.26(-5.34,-1.18)$ & .0025 \\
\hline & Former GXR patients & $53.32(50.75,55.89)$ & $-2.22(-4.97,0.53)$ & .1104 & $-1.52(-3.72,0.67)$ & .1703 \\
\hline & New patients & $59.98(56.74,63.22)$ & $-5.41(-7.60,-3.23)$ & $<.0001$ & $-4.53(-6.68,-2.37)$ & .0001 \\
\hline \multirow[t]{3}{*}{ Self-monitor } & Former placebo patients & $61.81(58.70,64.91)$ & $-6.39(-8.98,-3.79)$ & $<.0001$ & $-4.48(-6.45,-2.52)$ & $<.0001$ \\
\hline & Former GXR patients & $56.06(52.73,59.40)$ & $-4.93(-8.10,-1.76)$ & .0031 & $-4.23(-6.87,-1.58)$ & .0022 \\
\hline & New patients & $61.24(56.70,65.79)$ & $-7.86(-11.77,-3.96)$ & .0003 & $-6.23(-10.05,-2.40)$ & .0021 \\
\hline \multirow[t]{3}{*}{ Behavioral regulation index } & Former placebo patients & $63.09(60.46,65.73)$ & $-6.02(-9.06,-2.98)$ & .0002 & $-4.06(-6.05,-2.07)$ & .0001 \\
\hline & Former GXR patients & $56.39(53.43,59.35)$ & $-3.71(-6.52,-0.90)$ & .0109 & $-3.26(-5.52,-1.01)$ & .0053 \\
\hline & New patients & $64.73(61.24,68.23)$ & $-8.72(-11.53,-5.92)$ & $<.0001$ & $-7.98(-10.72,-5.23)$ & $<.0001$ \\
\hline \multirow[t]{3}{*}{ Initiate } & Former placebo patients & $68.51(65.65,71.38)$ & $-5.49(-8.51,-2.46)$ & .0006 & $-3.87(-5.94,-1.80)$ & .0004 \\
\hline & Former GXR patients & $59.71(56.58,62.84)$ & $-3.31(-6.64,0.02)$ & .0514 & $-2.03(-4.87,0.80)$ & .1563 \\
\hline & New patients & $69.10(64.97,73.22)$ & $-10.38(-13.98,-6.78)$ & $<.0001$ & $-8.95(-12.11,-5.79)$ & $<.0001$ \\
\hline \multirow[t]{3}{*}{ Working memory } & Former placebo patients & $73.91(71.07,76.75)$ & $-4.80(-8.45,-1.15)$ & .0111 & $-3.31(-5.68,-0.93)$ & .0069 \\
\hline & Former GXR patients & $66.18(62.57,69.78)$ & $-4.78(-8.24,-1.32)$ & .0079 & $-3.92(-6.70,-1.13)$ & .0066 \\
\hline & New patients & $74.10(70.29,77.91)$ & $-10.93(-14.98,-6.88)$ & $<.0001$ & $-10.15(-13.73,-6.57)$ & $<.0001$ \\
\hline \multirow[t]{3}{*}{ Plan/organize } & Former placebo patients & $70.51(67.74,73.28)$ & $-3.86(-6.94,-0.78)$ & .0152 & $-2.32(-4.37,-0.27)$ & .0270 \\
\hline & Former GXR patients & $63.02(59.52,66.51)$ & $-3.69(-7.09,-0.29)$ & .0340 & $-3.00(-5.90,-0.10)$ & .0426 \\
\hline & New patients & $70.51(66.57,74.45)$ & $-8.90(-12.63,-5.16)$ & $<.0001$ & $-8.75(-11.90,-5.60)$ & $<.0001$ \\
\hline \multirow[t]{3}{*}{ Task monitor } & Former placebo patients & $72.63(69.56,75.69)$ & $-6.76(-9.95,-3.56)$ & $<.0001$ & $-4.07(-6.35,-1.79)$ & .0006 \\
\hline & Former GXR patients & $63.85(60.22,67.49)$ & $-7.02(-11.06,-2.99)$ & .0011 & $-4.89(-8.34,-1.43)$ & .0063 \\
\hline & New patients & $0.71(66.07,75.34)$ & $-8.93(-13.17,-4.70)$ & .0002 & $-8.35(-12.12,-4.58)$ & $<.0001$ \\
\hline \multirow[t]{3}{*}{ Organization of materials } & Former placebo patients & $65.97(63.95,67.98)$ & $-5.00(-7.82,-2.18)$ & .0008 & $-3.24(-5.21,-1.26)$ & .0016 \\
\hline & Former GXR patients & $58.61(55.77,61.45)$ & $-3.31(-5.55,-1.08)$ & .0046 & $-2.87(-4.62,-1.11)$ & .0018 \\
\hline & New patients & $65.73(62.43,69.03)$ & $-8.41(-11.90,-4.93)$ & $<.0001$ & $-7.88(-11.03,-4.72)$ & $<.0001$ \\
\hline
\end{tabular}


Table 4 Key Efficacy Measures During Long-term Treatment With GXR (Continued)

\begin{tabular}{|c|c|c|c|c|c|c|}
\hline \multirow[b]{2}{*}{ Endpoint } & \multirow[b]{2}{*}{ Patient populations } & \multirow[b]{2}{*}{ Week 0} & \multicolumn{2}{|l|}{ Week 50} & \multicolumn{2}{|c|}{$\begin{array}{l}\text { Last observation in the } \\
\text { treatment period }\end{array}$} \\
\hline & & & $\begin{array}{l}\text { Change from } \\
\text { week } 0\end{array}$ & $p$-value & $\begin{array}{l}\text { Change from } \\
\text { week } 0\end{array}$ & $p$-value \\
\hline \multirow[t]{3}{*}{ Metacognition index } & Former placebo patients & $73.36(70.51,76.21)$ & $-5.80(-9.04,-2.55)$ & .0008 & $-3.75(-5.86,-1.65)$ & .0007 \\
\hline & Former GXR patients & $64.16(60.65,67.68)$ & $-5.02(-8.21,-1.83)$ & .0028 & $-3.82(-6.44,-1.20)$ & .0050 \\
\hline & New patients & $73.24(69.29,77.20)$ & $-11.14(-14.95,-7.33)$ & $<.0001$ & $-10.35(-13.69,-7.01)$ & $<.0001$ \\
\hline \multirow[t]{3}{*}{ GEC index } & Former placebo patients & $70.52(67.76,73.29)$ & $-6.41(-9.59,-3.22)$ & .0002 & $-4.22(-6.30,-2.15)$ & .0001 \\
\hline & Former GXR patients & $61.73(58.32,65.13)$ & $-4.84(-7.96,-1.73)$ & .0031 & $-3.90(-6.45,-1.36)$ & .0032 \\
\hline & New patients & $71.10(67.26,74.93)$ & $-10.86(-14.29,-7.43)$ & $<.0001$ & $-10.05(-13.21,-6.89)$ & $<.0001$ \\
\hline
\end{tabular}

AAQoL Adult ADHD Quality of Life Questionnaire, ADHD-RS-IV Attention-Deficit/Hyperactivity Disorder Rating Scale IV with Adult Prompts, BRIEF-A Behavior Rating Inventory of Executive Function-Adult Version, CAARS Conners' Adult ADHD Rating Scales, CGI-I Clinical Global Impression-Improvement, CGI-S Clinical Global Impression-Severity of Illness, Cl confidence interval, DSM-IV Diagnostic and Statistical Manual of Mental Disorders (Fourth Edition), GEC Global Executive Composite, GXR guanfacine extended-release, NA not applicable, PGI-I Patient Global Impression-Improvement

${ }^{a}$ Change from start of long-term treatment calculated using week 50 or last observation in the treatment period and assessed using two-sided $t$ tests

${ }^{b}$ Data are response rates at each time point. Differences in response rates from the start of long-term treatment or week 1 and week 50 or last observation in the treatment period were assessed using two-sided $t$ tests

${ }^{c}$ Data are response rates at week 1 of long-term treatment 